\title{
¿Qué educación física hemos vivido? Analizando nuestro saber profesional a partir de historias de vida
}

What kind of physical education have we received?

Analysys of our professional knowledge from experiences learnt by us

\author{
Victor M. López Pastor (coord.) \\ Roberto Monjas Aguado \\ Ana García Peñuela de Miguel \\ José M. García de La Puente \\ Marta González Pascual`
}

\section{Resumen}

Este ensayo intenta reconstruir cómo ha sido la educación física en nuestro pais en las décadas recientes. ¿Cuáles eran sus características principales y más habituales? ¿̨ué enfoques eran los más extendidos y cuáles eran los minoritarios? Dentro de lo posible, analizar y reflexionar sobre las razones de este estado de la cuestión y de las influencias acerca de cómo entendemos y practicamos en la actualidad la educación física. Para intentar reconstruir este intervalo temporal de nuestra historia más reciente, hemos recurrido a las fuentes orales de algunos de sus protagonistas principales: las personas que durante estas décadas han sido alumnos de primaria y secundaria ( $\mathrm{y}$ que por tanto lo fueron también de educación fisica), y que en la actualidad son profesores de educación física en formación inicial o e los primeros años de su práctica docente.

Palabras clave: Profesores, educación fisica, educación elemental, educación secundaria,

\section{abstract}

\begin{abstract}
This paper attempts to reconstruct how has been taught the physical education in our country recently. Which were the main, more usual qualities of the physical education? Which were the more extended approaches? Which were the less extended ones? If possible, this paper tries analyzing and thinking about the reasons of this subject and the influences about how the physical education is understood a practiced just now. To try reconstructing that period of time of our recent history we have recoursed to the main protagonists' oral sources: people that in their days have been pupils in elementary and secondary education, and at the present time they are physical education teachers.
\end{abstract}

key words: $\quad$ Teachers, physical education, elementary education, secondary education, main protagonists.

Fecha de recepción: 29 de julio de 2005.

Fecha de áceptación: 8 de agosto de 2005

Equipo de investigación. Centro: E.U. Magisterio Segovia. Universidad Valladolid Pza/ Colmenares, 1-40001 - Segovia (España) (tf: 921-11.22.99). 


\section{Las finalidades del estudio}

Iniciar procesos de análisis y reflexión sobre la EF vivida a durante la decada de ochenta y noventa. En el caso que nos ocupa, durante el paso por el sistema educativo como alumnado de primaria y secundaria.

Poner en común las historias de vida individuales, de forma que permitan iniciar procesos grupales de elaboración de un "mapa" o perfil de cómo era la EF en primaria en parte de nuestro país en la década de los ochenta y cuáles eran los enfoques predominantes a su paso por la enseñanza secundaria (BUP) en la década de los noventa.

-A través de la realización de este recorrido histórico (personal y colectivo), tomar conciencia de la historia más reciente de nuestra asignatura en nuestro contexto, por cuanto resulta ser un conocimiento fundamental a la hora de intentar comprender qué enfoques siguen siendo los predominantes en la escuela y por qué es así.

-Comprobar hasta que punto dichas experiencias están arraigadas en los planteamientos y creencias educativas del profesorado de EF y cómo influyen en su actuación docente.

En este sentido, consideramos conveniente iniciar procesos de reflexión e introspección que ayuden a tomar conciencia de:

a. Cómo se van configurando nuestras creencias sobre la EF;

b. Que la construcción de la EF es un proceso histórico y so cial en el que coexisten diferentes corrientes y enfoques,

aunque unas suele predominar sobre otras ${ }^{1}$, y cuáles han sido las que han configurado de forma predominante sus creencias sobre la EF.

Es fundamental comenzar a plantearse cuál es el valor educativo de dichas creencias y esquemas de pensamiento, y analizar y decidir si deberían ser modificados para adecuarse a nuestra profesión y a la legislación educativa vigente.

\section{Metodología utilizada para la realización del estudio}

\section{Técnicas e instrumentos de obtención de datos}

El instrumento fundamental de obtención de datos fue la historia de vida sobre la EF que este profesorado en formación inicial recibió durante su paso por el sistema educativo.
Los datos así fueron completados con entrevistas grupales y discusiones en grupo sobre las características que surgían en ese primer volcado de datos.

\section{Muestra del estudio}

La muestra la constituyeron alumnos de la especialidad de EF de cuatro promociones seguidas (cursos 1999, 2000, 2001 y 2002), que proviene de diferentes lugares de nuestra geografía nacional (Albacete, Castellón, Alicante, Canarias, Segovia, Ávila, León, Burgos, Soria, Ciudad Real, Madrid, Murcia, Navarra, Toledo, Zamora,...), aunque lógicamente la mayoría de ellos provienen de la zona centro del país.

\section{Análisis de datos}

A partir de los datos obtenidos con estas técnicas e instrumentos se crea un sistema de categorías que los engloba en su totalidad y permite su presentación en forma de resultados del estudio. Este primer sistema de categorías y resultados se somete a revisión y puesta en común con el mismo alumnado y a una posterior revisión crítica por parte de otros compañeros, así como a una contrastación con otros estudios similares, de modo que permita perfeccionarla y reorganizarla para su presentación a la comunidad científica.

\section{Sobre la validez externa y transferibilidad de los resultados}

Con este estudio no pretendemos generar leyes absolutas ni extraer conclusiones generales sobre lo que ha sido la EF en este periodo histórico, sino tan sólo recoger una primera muestra, un primer retazo sobre el mismo. Evidentemente de estos resultados no pueden establecerse generalizaciones por dos razones:

a. La primera es que los datos muestran que existían realidades muy diferentes entre unos centros y otros.

b. La segunda es que la finalidad de estos estudios tiene que ver más con la generación de procesos de reflexión y toma de conciencia con el profesorado, que con la realización de estudios con una elevada validez externa.

De todas formas consideramos que sus resultados pueden ser interesantes para la comunidad profesional, por cuanto aportan datos significativos sobre las características que la $\mathrm{EF}$ ha tenido en muchos centros durante una época reciente. Además, dichos datos permiten realizar un análisis de cómo dichas características influyen en la situación actual de nuestra materia y en su evolución presente y futura. 


\section{Revisión bibliográfica sobre esta metodología y tipo de estudio}

Existen muy pocos trabajos en nuestra área de conocimientos basados en las fuentes orales como vía de acceso y reconstrucción de nuestra historia profesional más reciente. En cambio, sí podemos encontrar trabajos sobre los conceptos previos que posee el profesorado de EF cuando comienza su formación inicial (Bores et al,1994; García Monge,1994; Martínez,1994; Torres et al,1995; Sicilia et al,1998;...) y también sobre la utilización de "Historias de vida" en la formación del profesorado de educación física(Tinning,1992,1994 Sparkes,1993,1994,1996; Pascual,1993,1994,2000; Devís y Sparkes, 1999, 2001; López et al, 2002;...).

\section{Sobre el interés de su utilización en la formación del profesorado de EF}

Nosotros entendemos que la utilización de las historias de vida en la formación Inicial del profesorado de educación física es una herramienta poderosa como técnica de introspección y toma de conciencia de sus creencias y planteamientos sobre educación física escolar; resultado especialmente interesante y útil en los procesos orientados a la formación reflexiva y crítica del profesorado ${ }^{2}$. $\mathrm{El}$ análisis colectivo en clase de la información extraída permite tomar conciencia de sus nuestras creencias previas sobre la profesión y hacerles nos conscientes de la necesidad de avanzar hacía modelos de EF más educativos.

\section{La educación Física que vivimos: características habituales y algunas exposiciones}

\section{Aclaraciones previas}

En este apartado presentamos las características encontradas al realizar el análisis conjunto de todos los datos recogidos. Recordamos que se trata de características principales de la EF que esta muestra de profesores en formación inicial ha vivido durante las dos últimas décadas, a su paso como alumnos por las etapas de primaria y secundaria. Evidentemente, los modelos $\mathrm{y}$ enfoques de $\mathrm{EF}$ que han vivido no son monocordes, sino que presentan cierta variedad. Esta visión global es sumamente interesante porque permite ir reconstruyendo la evolución de nuestra disciplina durante los últimos veinte años (cuáles eran los enfoques predominantes, cuáles los marginales y alternativos), así como comenzar a entrever cual ha sido su evolución en el tiempo y en qué medida dichos enfoques han ido modificándose, extendiendose o desapareciendo con el paso de los años.

\section{La EF que vivieron en los colegios de primaria duran- te la década de los ochenta}

La EF como un "recreo vigilado" ("deporte libre", "partidillo", "dejar balones" o "pachangita")

En un porcentaje muy alto de los casos estudiados, puede comprobarse como era una práctica muy extendida en primaria, tanto que en muchos casos era el proceder más cotidiano, cuando no el único. Esta forma de entender y practicar la EF suele ser el "enfoque" más habitual en la EF de las últimas dos décadas y, como posteriormente veremos, se mantiene también en secundaria, en muchos casos junto a la utilización de tests de condición física como modelo habitual de evaluación (calificación) y el trabajo de acondicionamiento físico y de entrenamiento deportivo.

\section{La clara separación por sexos}

Nuestro alumnado ha vivido una EF en la que la separación por sexos constituía la nota predominante. Lo cual, en algunas ocasiones lo cual, incluía también la existencia de profesores y profesoras (y programas) distintos para cada sexo, mientras que en otras la separación era por actividades (los chicos a jugar fútbol, baloncesto o balonmano y las chicas a hacer bailes, gimnasia rítmica y/o a jugar voleibol).

\section{$L$ L variedad de horarios y carga docente de la EF}

En lo que se refiere a la cantidad de horas semanales dedicadas a la EF y su organización en el horario, las experiencias de nuestro alumnado varían entre un 10 $\%$ que prácticamente no hacía nunca EF y otro $10 \%$ que cumplía de manera sistemática las dos horas que tenían asignadas oficialmente. Entre ambos extremos existían abundantes casos de una única hora semanal, o la dependencia de factores diversos (hacer buen o mal tiempo, haberse portado bien, terminar las tareas a tiempo,...) que influían en dedicar más o menos tiempo a la EF.

Aún hoy en día hay muchos centros (en especial privados), que no imparten las tres horas semanales de EF que por ley corresponden a esta etapa, o en los que la impartición de una o dos de dichas horas sigue dependiendo de los mismos factores de hace veinte años (ganas o no del maestro-tutor de impartirla, comportamiento del alumnado, cuestiones climáticas, el retraso que se lleve en otras materias, etc.). 
Los contenidos de la EF

En los casos en que el profesorado desarrolla un programa concreto (poco más de la mitad), los contenidos que se repiten con mayor asiduidad son los de: iniciación deportiva, habilidades gimnásticas y condición física; a continuación: juegos, correr, gimnasia sueca y premilitar, deportes concretos (baloncesto, voleibol, atletismo, balonmano, fútbol). Por último, la expresión corporal sólo aparece en un caso.

En muchos de estos casos, todos los años se desarrollan los mismos contenidos (independientemente del curso o etapa) y la "iniciación deportiva" suele plantearse desde enfoques de entrenamiento y competición.

\section{La evaluación}

En la inmensa mayoría de los casos la evaluación en EF se basaba en la aplicación de tests de condición física $\mathrm{y}$, a veces, también en tests de ejecución de habilidades deportivas; independientemente de que se hubieran trabajado o no dichos contenidos durante el periodo correspondiente.

Esta curiosa y grave incoherencia entre las actividades que se desarrollan de manera habitual en las clases de EF y la forma, métodos y criterios con que se (de)evalúa(califica) la asignatura, es un reflejo más. de las múltiples incoherencias educativas que durante décadas ha mostrado nuestra área, y que justifican su bajo estatus educativo (véase Rivera y Trigueros,1999; López,1999).

En los casos en que la finalidad principal o única de la EF era el trabajo de acondicionamiento físico y entrenamiento deportivo; la utilización de este tipo de pruebas de evaluación guarda al menos cierta coherencia con las finalidades y los contenidos trabajados.

La estructura de las sesiones

En las escasas ocasiones en que existía, se basaba en la habitual estructura de: calentamiento, parte principal y vuelta a la calma; más propia de sesiones de entrenamiento físico-deportivo que de prácticas educativas. Hemos analizado en detalle los tipos de estructura de sesión en EF y sus implicaciones educativas en un trabajo anterior (véase López et al, 2001).

El juego y lo lúdico como símbolo de progreso, avance, innovación y profesionalidad en $E F$

En algunos casos el alumnado realizaba las clases a través de juegos, lo cual era considerado como clara muestra de un importante avance profesional y de haber vivido una EF de una calidad educativa mucho mayor que la del resto de sus compañeros.
Durante los últimos años puede comprobarse una peligrosa tendencia a considerar que ser profesor de EF consiste, básicamente, en dominar un conjunto de juegos con los que trabajar todos los contenidos de la EF. En las últimas décadas el "juego" se ha convertido en una especie de "piedra filosofal" de la profesión, en el remedio adecuado para todo tipo de contenidos, objetivos y finalidades. El juego vale para todo y todo se soluciona con un juego. Además el juego y lo lúdico parecen ser el símbolo de progreso, avance, innovación y profesionalidad en EF; la diferencia entre la EF tradicional y la educativa (y entre los antiguos profesores y los actuales) parece ser fundamentalmente la utilización (o no) del juego para trabajar los correspondientes contenidos (que, en cambio, no se cuestionan demasiado). En un trabajo reciente (López y otros, 2003) analizamos con más profundidad esta cuestión ${ }^{3}$.

La progresiva deportivización y secundarización de la EF en primaria

En los datos recogidos se observa que no existen demasiadas diferencias entre los contenidos que se enseñan en el área de $\mathrm{EF}$ en primaria con respecto a los que se enseñan en secundaria; tampoco parecen existir demasiadas diferencias en cuanto a la forma de evaluarcalificar.

\section{La EF que vivieron en secundaria durante la década de los noventa}

\section{La EF como entrenamiento físico}

Las clases de EF pasan a convertirse, fundamentalmente, en un trabajo (entrenamiento) de la condición física del alumnado. De hecho, en varios casos las clases se dedicaban a preparar los tests de condición física con los que serían "evaluados" (calificados) al final del trimestre.

\section{El "deporte libre"}

Continúa siendo bastante habitual, aunque ya no en un grado tan alto como en la etapa y década anteriores.

\section{Los contenidos}

Cuando hay un programa más o menos estructurado, los contenidos se centran en la condición física, las habilidades gimnásticas y los deportes (predominan voleibol, baloncesto y balonmano; seguidos del atletismo y el fútbol; sólo en un caso se cita la gimnasia rítmica, el frontenis y la esgrima). La expresión corporal ni siquiera aparece. En algunos de estos casos todos los años se desarrollan 
los mismos contenidos, y la "iniciación deportiva" continúa planteándose desde enfoques de entrenamiento y competición.

\section{La evaluación-calificación}

En la inmensa mayoría de los casos se reduce a la aplicación de tests de condición física; bien al principio y final de cada curso, bien al final de cada trimestre. En algunos casos también se examina sobre las habilidades gimnásticas o deportivas. Unas veces la calificación se obtiene directamente de los resultados de los tests, y otras se tabula con valoraciones sobre la participación y el esfuerzo ${ }^{4}$. A continuación presentamos un cita sacada de una historia de vida, que hace referencia al predominio de este tipo de sistemas de evaluación-calificación (y sus implicaciones):

Como alumna nunca tuve problemas en EF, porque era deportista y estaba acostumbrada al tipo de evaluación deportiva o de rendimiento; es más, la prefería porque se me daba mejor. Pero nunca fui consciente de aprender nada en $\mathrm{EF}$. $\left(\mathrm{H}^{\circ}\right.$ de Vida; octubre 2002 , alumna $3^{\circ}$ magisterio).

\section{La irrupción de la "teoría"}

Las clases "teóricas" (y sus correspondientes exámenes) comienzan a ser más habituales, utilizándose para muchas de ellas el libro de texto, fotocopias elaboradas por el profesoro sacadas de algún libro y videos (el alumnado debe tomar apuntes de la información del video y estudiarlos para el examen ${ }^{5}$ ). Los contenidos a estudiar y examinar no varían demasiado entre unos casos y otros: acondicionamiento físico, anatomía, reglamentación, lesiones y técnica y táctica deportiva. También se dan casos en que sólo los lesionados o enfermos deben realizar "trabajos" sobre alguna de estas temáticas.

\section{Metodología y separación por sexos}

Las metodología es predominantemente directiva. En lo referente a la diferenciación de actividades en función del sexo del alumnado, todavía se mantiene en algunos casos (entre el 15 y el 20\%, según el grupo).

\section{La experiencia personal en $E F$}

Continúan apareciendo bastantes valoraciones positivas sobre sus experiencias respecto al deporte y la EF en esta época, pero también existen casos en que sus vivencias de la $\mathrm{EF}$ durante el instituto comenzaron a ser negativas, e incluso explican cómo fueron aprendiendo estrategias de evasión durante sus clases de EF. Dados los enfoques de EF predominantes, las experiencias negativas comienzan a ser mucho más numerosas entre las alumnas que entre los alumnos.

\section{Conclusiones finales: Algunos efectos en los modelos de educación física que tenemos interiorizados e implicaciones para la formación del profesorado}

Las formas de entender y practicar la EF que vivimos como alumnos, junto a las que hemos visto hacer durante nuestras primeras experiencias como profesores, suele influir en nuestras creencias y modelos de EF bastante más de lo que solemos pensar. También suelen marcar nuestras prácticas como profesores actuales de $\mathrm{EF}$ de una forma mucho más poderosa de lo que la mayoría de nosotros somos conscientes.

Lógicamente, las creencias más asentadas de este profesorado en formación sobre las características de la EF que van a impartir cuando accedan a la práctica docente pueden resumirse en los conceptos denominados: "deportivización y secundarización de la EF”. Esto es, una gran parte de nuestro alumnado conoce y defiende una EF basada en la condición física y el deporte, desde una óptica de entrenamiento y rendimiento. Ven al profesor como un entrenador y como un monitor que pasa tests cada cierto tiempo (eso sí, un entrenamiento muy "lúdico", todo a través de "juegos",...).

En este sentido, la evolución que parece mostrar la profesión de una forma mayoritaria es avanzar hacia la utilización del "juego" como metodología principal o única, con la cual continuar trabajando los mismos contenidos y desde similares enfoques y planteamientos; o bien desde enfoques puramente recreativos ("lo que importa es que se lo pasen bien").

En cualquier caso parece obvio que muchas de las razones del bajo estatus que tiene nuestra materia nos las hemos ganado a pulso (la EF como un recreo más partidillos, tiempo libre, recreacionismo; la permisividad en el incumplimiento de las horas designadas para el área; la utilización del área como premio o castigo para el comportamiento en otras materias, o para el desfogue entre ellas; la utilización de sistemas de evaluación-calificación inadecuados; el sexismo; la ausencia de programaciones sistemáticas y el "todo vale"; la reiteración de contenidos;...).

También parece algo obvio que esta profesión ha avanzado de modo notable en la última década, y que hay miles de compañeras y compañeros bastante com- 
prometidos en construir día a día una materia educativamente valiosa, que va superando poco a poco todas las limitaciones con que partía, y desarrollando el enor- me potencial educativo que encierra. Ese es también nuestro propósito, y esperamos que este trabajo pueda aportar algo en ese sentido.

\section{Bibliografía}

BLÁZQUEZ. (2001). La Educación Física. Barcelona. Inde.

BORES CALLE, N. y otros (1994). Análisis de las concepciones de educación física de los alumnos como instrumento para transformar la práctica docente. Actas XII Congreso Nacional Educación Fisica, Sevilla. Wanceulen. (35-41). E.U. Magisterio

DEVÍS, J. y SPARKES, A. (1999). Burning The Book: A Biographical Study Of A Pedagogically Inspired Identity Crisis In Physical Education. En European Physical Education Review, Vol 5, No.2 (135-152).

(2001). Quemar el libro. En Devís, J. (coord...) La educación fisica en el siglo XXI". Ed. Marfil. Alcoy.

GARCIA MONGE, A. (1994). Ideas dominantes del alumnado de la especialidad de educación física de Palencia sobre el juego. Análisis y tratamiento. Actas XII Congreso Nacional Educación Física, Sevilla. Wanceulen. E.U. Magisterio y I Fac. Educación.

HERNÁNDEZ ÁLVAREZ, J. L.(2000). El futuro de la formación inicial del profesor de educación fisica. En Actas XVIII Congreso Nacional Educación Fisica. Ciudad Real. Universidad de CastillaLa Mancha. Cuenca (95-110). E.U. Magisterio.

LÓPEZ PASTOR, V. M. (1999). Prácticas de evaluación en educación física: estudio de casos en primaria, secundaria y formación del profesorado Valladolid. Ed. Universidad de Valladolid.

LÓPEZ PASTOR, V. M. (coord.) (1999). Educación física, evaluación y reforma. Segovia. Librería Diagonal.

LÓPEZ, V. (2000). La evaluación en educación física en España: ună revişión bibliográfica (1970-1997). Rev. Habilidad Motriz, 16 (2236). Córdoba. COPLEF de Andalucia.

LÓPEZ, V. et al (2001). La sesión en educación fisica: los diferentes modelos y los planteamientos educativos que subyacen. En Rev. Lecturas de educación fisica y deportes, 43. (www.efdeportes. com).

LÓPEZ, V. M. y otros (2001 b). Las nuevas tecnologías en la formación del profesorado de educación física (I):análisis crítico de la situación actual. En La enseñanza de la educación física y el deporte escolar. Cantabria. Ed. ADEF.

(2002). La utilización de historias de vida en la formación inicial del profesorado de educación física. Inédito.

(2003). Cuestionando tópicos en educación física: La estructura de sesión y la utilización de los juegos como recurso didáctico. En Revista La red, No. 5 (40-42). Badajoz. Ed. Amefex.

MATEOS CARRERAS, M.J. (1998). La EF en primaria o los juegos. Actas XVI C.N. de EF de EUM. Badajoz. (pp. 243-246). Universidad de Extremadura.

\section{Notas}

1 Sobre el proceso histórico de construcción del actual currículum del área de EF y las corrientes que influyen en su elaboración, consultar el trabajo de Hernández (1996). Una revisión de las diferentes corrientes históricas de la EF, su evolución e influencia en la EF actual y el futuro de la misma puede encontrarse en los recientes trabajos de Zagalaz (2001) y Blázquez (2001)

2 Hemos desarrollado este tema con mayor profundidad en un reciente trabajo (López et al.,2002).

3 Sobre esta temática aconsejamos consultar el trabajo de Mateos Carreras (1998).

4 Sobre el tema de la evaluación en EF y su evolución histórica en los últimos treinta años ver nuestros trabajos anteriores (López,1999,2000).

5 Sobre este tipo de usos de las nuevas tecnologías en EF consultar López y otros (2001). física. Actas XII Congreso Nacional Educación Física, Sevilla. Wanceulen. E.U.Magisterio y I Facultades de Educación.

PASCUAL BAÑOS, C. (1993). La evaluación de un programa de educación fisica para la formación inicial del profesorado: algunos problemas, dilemas y/o contradicciones". Actas II Encuentro Unisport de Sociología Deportiva (pp. 183-202). Málaga. Unisport.

(1994). La evaluación de un programa de educación fisica para la formación inicial del profesorado: un estudio de caso. Tesis Doctoral. Universidad de Valencia.

(2000). La pedagogía crítica, una cuestión de ética. Actas XVIII Congreso Nacional de Educación Física de Escuelas de Magisterio. Ciudad Real. Cuenca (pp. 73-93). Universidad de Castilla-La Mancha.

RIVERA, E. y TRIGUEROS, C. (1999). Educación fisica: ¿la chacha del 2001 en educación primaria. En Conceptos, No. 6 La EF en la escuela (pp. 33-46). Granada. Ed. Alhama.

SICILIA CAMACHO, A. et al. (1998). Perfil del alumnado de educación física universitario. El caso de Almería. Actas XVI Congreso Nacional de Educación Física de Escuelas de Magisterio. Badajoz. Universidad de Extremadura. (pp. 47-64).

SPARKES, A. (1993). Experimentando una triple marginación en educación física. Perspectivas, 13 (pp. 2-7). León. INEF.

(1994). Problemas éticos de la investigación de paradigmas alternativos: de la dinámica del compromiso a la política de la representación. Actas II Encuentro UNISPORT de Sociologia Deportiva (73-92). Málaga. Unisport.

(1996). Recordando los cuerpos de los profesores: momentos desde el mundo de la educación física. Revista de Educación, 311 (pp. 101-118). Madrid. MEC.

TINNING, R. (1992). Educación física: la escuela y sus profesores. Valencia. Universidad de Valencia.

Reflexionando sobre nuestra práctica: enseñanza reflexiva, Investigación-acción y formación del profesorado de educación física. En Perspectivas, 15 (44-49). León. INEF.

TORRE NAVARRO, E. y otros. (1995). Conceptos, valores, motivaciones y experiencias sobre el deporte en los futuros maestros especialistas en EF de facultad de educación de Granada. Actas XIII Congreso Nacional de Educación Física (491-493). Universidad de Zaragoza E.U. Magisterio y Facultades Educación.

ZAGALAZ, M. L. (2001). Corrientes y tendencias de la educación física. Barcelona. Inde. 